\title{
All Regeneration for Optical Communication Network Using Optical 3R Regeneration and Phase sensitive amplifier
}

\author{
Bhagwan Das $^{1}$, M.F.L Abdullah ${ }^{1}$, Mohd Shah Nor Shahida ${ }^{1}$ and Qadir Bukhsh ${ }^{1}$ \\ ${ }^{1}$ Universiti Tun Hussein Onn Malaysia (UTHM) \\ he130092@siswa.uthm.edu.my
}

\begin{abstract}
Nonlinear optical effects in fibers occur via ultrafast Kerr nonlinearity, offers a flexible framework within which numerous signal-processing functions can be accomplished. When high power launched in optical fiber, several nonlinear transmission impairment such as amplitude noise, phase noise, power spectral losses, that degrades the performance of optical communication system. All optical regeneration is one of the solution to mitigate transmission impairments instead of optical to electrical conversion. In this paper, all optical regeneration is demonstrated for 10Gb/s DPSK system using $3 R$ regeneration and Phase sensitive amplification to mitigate amplitude and nonlinear phase noise form $10 G$ noisy DPSK transmission system. Bit error rate of $10^{-11}$ is achieved at power penalty of $6 \mathrm{~dB}$. The system is developed and tested using optisystem. The developed all optical regeneration system is very demanding for long distance transmission in high-speed communication systems.
\end{abstract}

Keywords: DPSK system; Optical regeneration; Phase sensitive amplifier; $3 R$ regeneration; Bit Error Rate

\section{Introduction}

In optical transmission systems a great part of the research is focused on increasing the system reach and robustness [1]. All-optical signal regeneration is auspicious techniques to achieve this extension, which can be performing with fiber nonlinearity. Optical nonlinear material is specially used for ultra-high speed performance. Power launched inside the optical fiber interacts with fiber material and due to refractive index several transmission impairments for example linear, nonlinear, scattering and etc. are produced [2]. These interactions depend on the noise evolution of signals transmitted inside the fiber[3-4]. Practically nonlinear phase noise is much more dominant than amplitude noise. During system design nonlinear phase noise is dealt separately [5, 6]. Regeneration signals are based on (electronic or all-optical signal) processing both are significantly in use depends upon application. Electronic-based regeneration requires conversion of data by mean of optical-to-electrical- ]optical [7, 8]. All optical regeneration do not require any conversion O-E-O [8]. An ideal regenerator would suppress noise in both the signal's amplitude and phase $[9,10]$. However, use of the complex optical-field gives rise to a new dominant limitation to system performance, namely nonlinear phase noise [13-14].

\section{Literature Review}

Phase sensitive amplification is one of the promising and powerful techniques that are used for all optical regeneration for both phase and amplitude regeneration [15-18]. All optical regeneration using previously investigate through single pump configuration (Non-degenerated) [1-3], dual pump configuration (degenerated) using Phase sensitive amplifier (PSA) for single or multilevel optical regeneration each has its own advantages 
and disadvantages[4-9]. Furthermore the all optical regenerations are also investigated theoretically, numerically with $2 \mathrm{R}$ (Re-amplify, reshaping) regenerator configuration, 3R (Re-amplify, reshaping, retiming), phase extraction, pump dithering, Semiconductor optical amplifier (SOA), format conversion and with black box model [9-13], [15]. To date with wide usage of above PSAs configuration and $2 \mathrm{R}$ regeneration is power consumptive and complex to use [16-18]. All optical regeneration still requires consideration to improve existing techniques in terms of improvement in BER, Eye diagram, power consumption and most important need to be energy efficient because not all developed techniques are energy efficient yet. To improve the performance of existing all optical regenerative systems, It is demonstrated that all optical regeneration using 3R and PSA, which has improved BER, reduces the both amplitude and phase noise from $10 \mathrm{G}$ DPSK system.

\section{Problem Statement}

When intense power is transmitted in fiber, nonlinear parameters become more dominate to degrade the signal and limits performance of optical communication system. This degradation produces following affects; loss in power spectral efficiency due to phase noise produces in co-propagating channels interacts with each other. This becomes severer in performance degradation when increase in transmission speed and distance. In this paper, all optical regeneration is demonstrated for DPSK system, using 3R regenerator and PSA techniques that will use to mitigate the ASE noise and nonlinear phase noise respectively. This technique gives the significant improvement in BER for 10G noisy DPSK system.

\section{Methodology}

All optical regeneration is performed for 10G DPSK systems using PSA with 3R regeneration. With this technique the nonlinear phase noise mitigate with PSA and other noises such as ASE are removed using $3 \mathrm{R}$ regeneration process. Figure 1. Shows the system model for the all optical regeneration system using 3R and PSA technique. The model contains the optical transmitter of $10 \mathrm{G}$ DPSK system. Noise emulator introduces the external noise in the channel to perform the regeneration. After that, all optical regeneration is performed for $10 \mathrm{G}$ DPSK system. At the end BER is compared before and after applying our technique for all optical regeneration system.

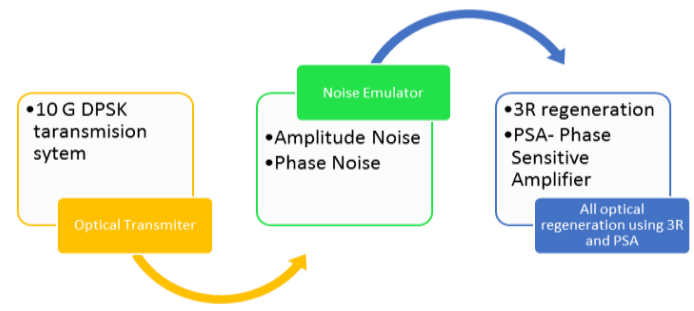

Figure 1: System Model

\subsection{Optical Transmitter}

The ability to signal in phase as well amplitude has long been exploited in telecommunications to boost spectral efficiency. In fiber optic communication, PSK format such as differential phase shift keying (DPSK) and differential quadrature PSK (DQPSK) offers significant benefits over amplitude only formats such as on-off keying 
(OOK). DPSK optical transmitter is to be design for this particular system because it offers advantage in terms of resilience to transmission impairments such as dispersion [3-7], and nonlinearity, as well as an inherent $3 \mathrm{~dB}$ improvement in receiver sensitivity over other amplitude formats with sophisticated error correction techniques and system margin improvements reach are enable. Equation (1) describes the mathematical expression for the DPSK optical transmitter, which states that can transmit different amount of power $\mathrm{P}(\mathrm{t})$, depending upon the encoding scheme and sampling time Ts.

$$
P(t)=\left(\begin{array}{c}
E \cdot \text { for } R Z 100 \% \\
E * \cos \left(\frac{\pi}{2} \cos ^{2}\left(\frac{1.5 \pi t}{T s}\right)\right) \cdot \text { for } R Z .66 \% \\
E * \cos \left(\frac{\pi}{2} \cos ^{2}\left(\frac{3 \pi t}{T s}\right)\right) \cdot \text { for } R Z .50 \%
\end{array}\right)
$$

The DPSK transmitter requires the following components to be design in Optisystem. Pseudo random bit sequence, laser source, pulse generator and optical modulator. Table 1 Shows, the parameters used in optical transmitter.

Table 1: Parameters for DPSK Transmitter

\begin{tabular}{|c|l|l|}
\hline \multirow{3}{*}{$\begin{array}{c}\text { Pseudo Noise } \\
\text { Random Sequence }\end{array}$} & Bit Rate & $10 \mathrm{G}$ \\
\cline { 2 - 3 } & Operation mode & Log sequence with Mark Probability of 0.5 \\
\cline { 2 - 3 } & Number of leading zeros & (Time window * $3 / 100) *$ Bit rate \\
\hline \multirow{2}{*}{ RZ pulse generator } & Pulse shape & Rectangular with 1Vp-p \\
\cline { 2 - 3 } $\begin{array}{c}\text { Continuous Wave } \\
\text { Laser }\end{array}$ & Encoding Scheme with Duty Cycle & RZ format 50\% \\
\cline { 2 - 3 } & Frequency & $1552.5 \mathrm{~nm}$ \\
\cline { 2 - 3 } & Power & $10 \mathrm{dbm}$ \\
\cline { 2 - 3 } & Linewidth & $10 \mathrm{MHz}$ \\
\cline { 2 - 3 } & Initial Phase & 0 \\
\hline \multirow{2}{*}{ Modulator } & Extinction ratio & $30 \mathrm{~dB}$ \\
\cline { 2 - 3 } & Negative signal chirp & 0 \\
\cline { 2 - 3 } & Symmetry factor & -1 \\
\hline
\end{tabular}

Figure 2 shows the DPSK transmitter designed in Optisystem. The systems contains Pseudo noise random sequence, $\mathrm{CW}$ laser as the source and $\mathrm{RZ}$ pulse generator as an electrical input. All these signals feed in to $\mathrm{LiNbO}_{3}$ MZIMs modulator, which produces the 10G DPSK output. The optical transmitter also consists of narrow-line width laser to generate a lightwave whose wavelength conforms to the ITU grid.

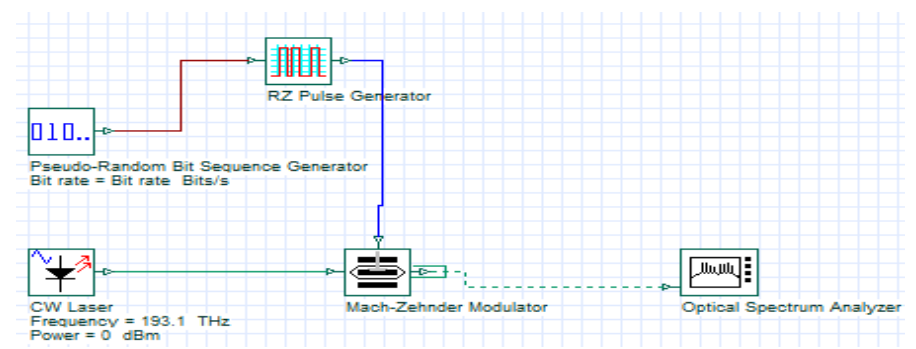

Figure 2: PSK Optical Transmitter in Optisystem 


\subsection{Noise Emulator}

In order to emulate the noise effects in the 10G DPSK transmitter, the amplitude and phase modulator are used. The phase modulator driven at a frequency of $20 \mathrm{GHz}$ and amplitude modulator driven at frequency of $1 \mathrm{GHz}$, both modulators are shown in Figure 3 . The amplitude and phase is controlled by varying the corresponding depths to emulate different noise levels allowing control of the phase and amplitude noise levels, respectively. The distorted signal will be launching in all optical regeneration system using $3 \mathrm{R}$ and PSA.

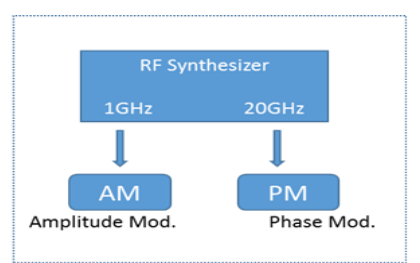

Figure 3: Model of Noise Emulator

Figure 4 displays, the noise emulator design in optisystem. The amplitude modulator is modulated with $1 \mathrm{GHz}$ sine wave frequency and phase modulator is modulated with 20 $\mathrm{GHz}$ sine wave. Both the modulator contains the sine generator as source to drive the modulator. The amplitude modulator includes $1 \mathrm{Vp}-\mathrm{p}$ amplitude and phase shift of $90^{\circ}$ and having modulation index of 1 . The Phase modulator includes the 1Vp-p amplitude, bias of 0.5 and phase shift of $90^{\circ}$, having normalized electrical signal with phase deviation of $90^{\circ}$. Overall, $12 \mathrm{~dB}$ noise is included in 10G DPSK system using both modulators.

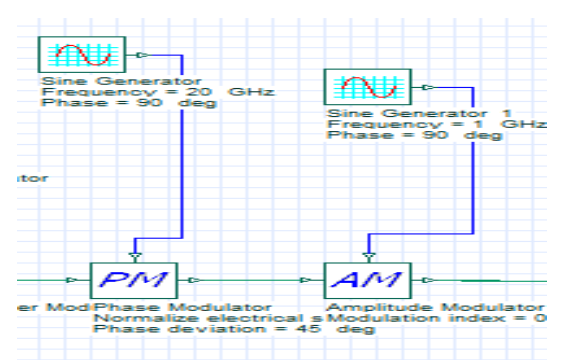

Figure 4: Noise Emulator In Optisystem

\section{3. $3 R$ regenerator and Phase sensitive Amplifier}

Devices that perform reamplification, reshaping and retiming is called $3 \mathrm{R}$ regenerators. If neglecting dispersion, only phase is affected by SPM:

$$
U(L, t)=u(O, t) \exp \left[i \gamma P L|u(O, t)|^{2}\right]
$$

Regenerator performance depends mainly on parameter.

Optimum value of $\phi \mathrm{nl}$ is close to $3 \pi / 2$ because spectrufe,then exfibits two peaks with a sharp dip at the original wavelength. The main interest of $3 \mathrm{R}$ optical regeneration is to prevent from noise accumulation during transmission. BER of signal accumulation scales are shown in equation 2 and 3 as:

$$
B E R \approx \exp \left(-C \frac{S N R}{N}\right)
$$

For 3R regenerator

$$
B E R \approx N \exp (-C S N R)
$$


SNR is the signal to noise ratio, $\mathrm{N}$ is the rank of the regenerator and $\mathrm{C}$ a suitable constant. One can obviously see the advantage of avoiding noise accumulation with a regenerator. Phase change of pump from $\phi=0$ to $\phi=\pi$ in equation 5 corresponds to shifting the modulation by $1 / 2$ pump period, which is equivalent to $1 / 4$ signal period. In one quadrature amplitude of the signal wave corresponding to the $\phi=0$, signal is amplified by a gain of $2^{\alpha}$ but in second quadrature corresponding to $\phi=\pi$ amplitude is deamplified $2^{\alpha^{\prime}}$. This is called a PSA as shown in equation 5 , the two quadrature amplitudes are a and $b$;

$$
E=a \cos (\omega t+\phi)+b \sin (\omega t+\phi)
$$

and phase of the pump signal is to be amplify the cos(wt) component and deamplify the $\sin (\mathrm{wt})$ component, the two-kinds of input signals with isotropic (phase insensitive) noise are transformed to the squeezed state as shown in Figure 5. When the input noise is dependent on thermal noise, the process is called thermal noise squeezing.

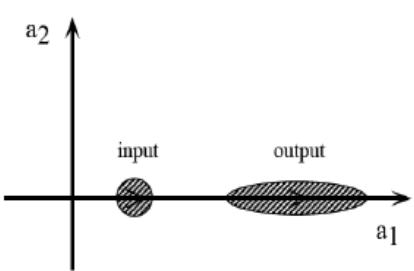

(a)

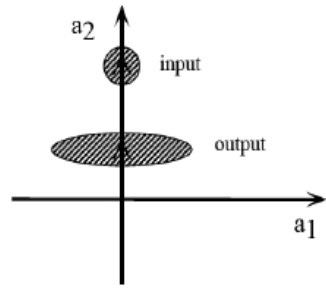

(b)

Figure 5: Phase Sensitive Amplifier Output

\subsection{All Optical Regenerator Using 3R Regenerator And Phase Sensitive Amplifier}

All optical regeneration is performed using 3R and PSA technique. Figure 6 illustrates the model for the all optical regeneration in Optisystem. The algorithm to performed all optical regeneration is developed in Matlab.

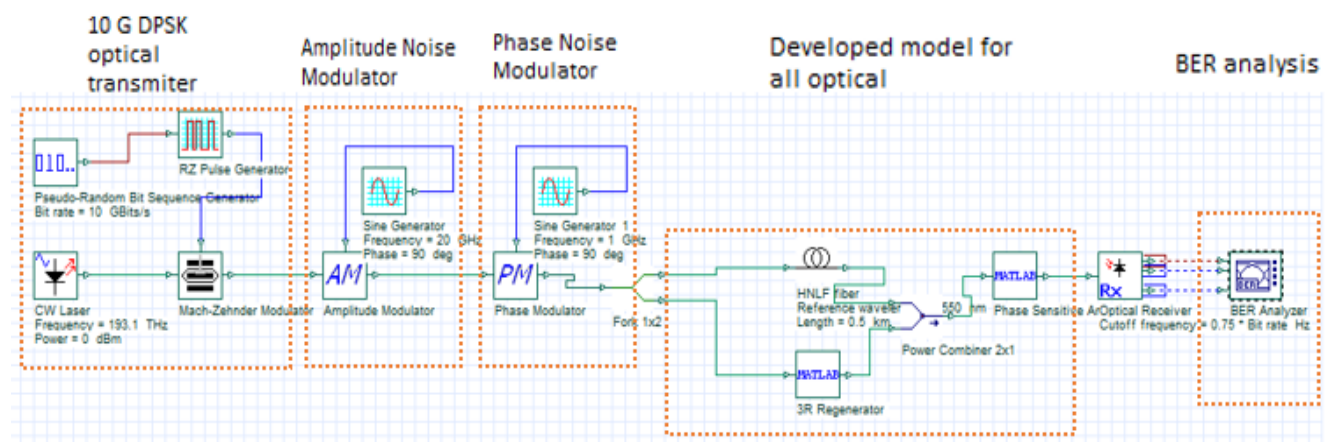

\section{Figure 6: Developed Model For All Optical Regeneration Using 3R And PSA}

Figure 6 states that first 10G DPSK signal is generated, after that amplitude and phase is included in the system externally. The all optical regeneration is performed over this noisy signal using developed algorithm (all optical regeneration algorithm using 3R and PSA). After that, BER is calculated before and after the all optical regeneration to verify the algorithm functionality. In all optical regeneration algorithm using 3R and PSA, 3R regenerator only can mitigate the ASE noise, synchronize the pulses, recover the signal 
for amplitude regeneration not for phase as much efficiently, overall 3R regeneration does not improve the BER of the optical system. To mitigate the nonlinear phase noise and to improve the BER of the optical system using $3 \mathrm{R}$ and PSA together. The main advantage of this technique is that with the help 3R regenerator the ASE noise and other amplitude related noise can mitigate and PSA will control the phase response of the system. Finally, all-optical regeneration is achieved using the 3R and PSA, with improved BER.

\section{Results and Discussion}

The optical DPSK transmitter transmits $10 \mathrm{G}$ pseudo noise sequence with modulated wave of CW laser of $1552 \mathrm{~nm}$. Figure 7 displays, the response of optical transmitter in optical spectrum analyzer. The global parameter for the model are setup as sample bit 64, sample rate $60 \mathrm{KHz}$, sample length 128 with Bit rate of $10 \mathrm{~Gb} / \mathrm{s}$.

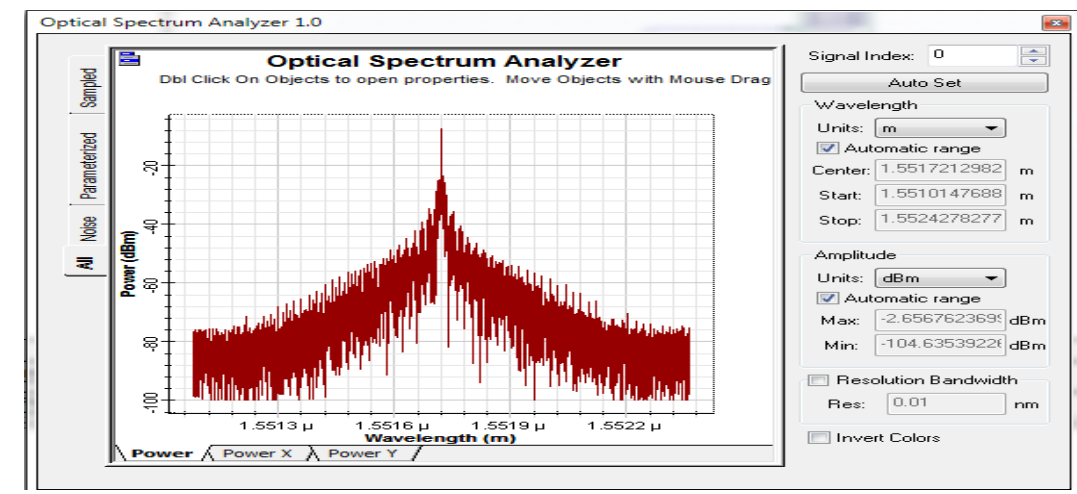

Figure 7: Response of DPSK Transmitter

Figure 8 and Figure 9 shows, response of noise emulator includes amplitude noise modulator and phase noise modulator respectively using optical spectrum analyzer using Optisystem, after inserting noise in 10G DPSK input.

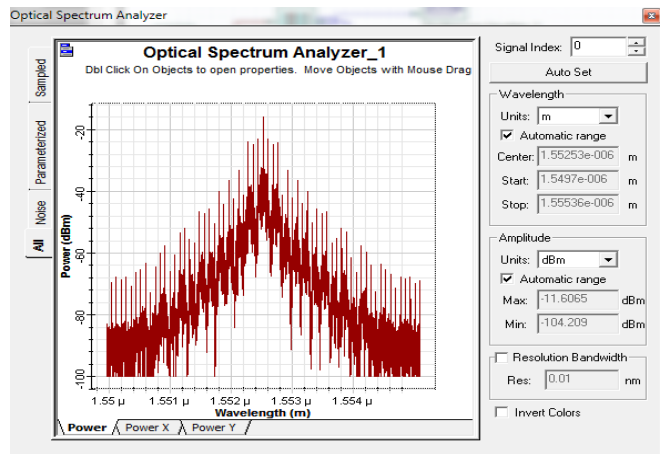

Figure 8: Response Of DPSK Transmitter Before Including Amplitude Noise And Phase Noise

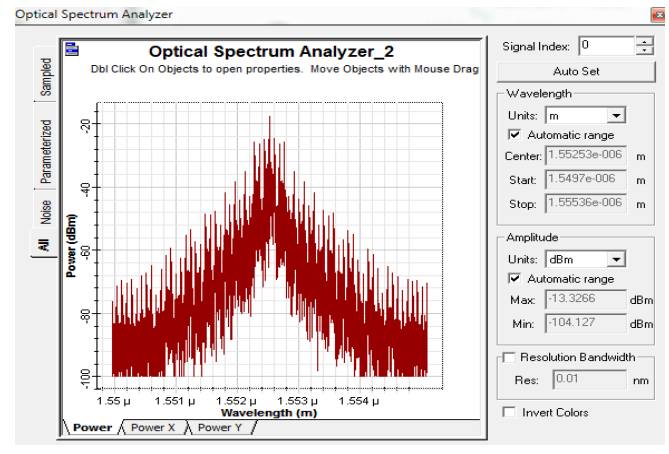

Figure 9: Response Of DPSK Transmitter After Including Amplitude Noise And Phase Noise

The ideal BER should be $10^{-15}$ for $10 \mathrm{G}$ DPSK system and ideally eye diagram should be opened and having no jitters. Figure 10 describes, the BER and eye diagram before the all optical regeneration, the BER at this level is $10^{-4}$, and eye diagram is also not eye opened and having jitters and in this case the Q-factor for the system 38. Using our 
developed techniques, It has been noted that BER up to $10^{-11}$ is improved, with eyeopened diagram and Q-factor achieved for the system is 118 as shown in Figure 11. The results sates that, achieved the significant improvement in BER and 68\% improvement in the Q-factor of the system with eye opened diagram, having minimum jitters. The remaining noise in the system is $4.5 \mathrm{~dB}$; this states that that all optical regeneration has removed the noise from the system significantly. All these results are achieved at modulation index of one. The less modulation index will also affects in increasing the BER. It isS highlighted this problem during our findings; in future, there is a need to design such system that are independent from this modulation order.

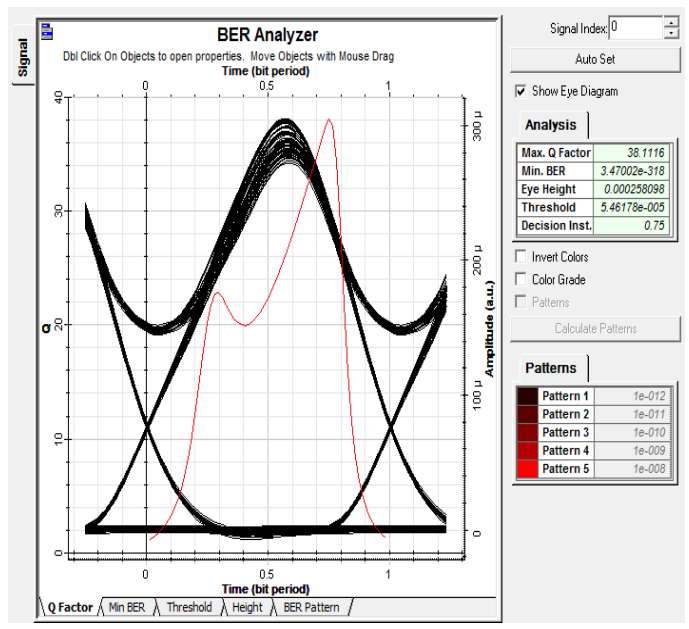

Figure 10: BER Before Applying All Optical Regeneration Using 3R And PSA

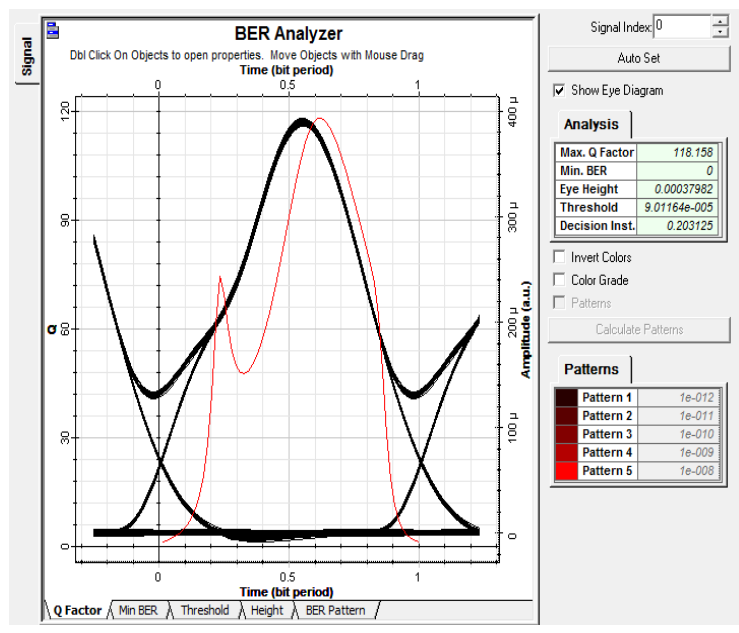

Figure 11: BER After Applying All Optical Regeneration Using 3R And PSA

\section{Conclusion}

The result of this study concludes that all-optical regeneration using 3R and PSA technique is feasible to use for 10G DPSK system that provides significant improvement in BER and Q-factor of the system. The result of this study suggest a number of new avenues for research, such as using similar technique $3 \mathrm{R}$ and PSA, the regeneration can be performed for PSK, DQPSK in high speed optical communication system. Furthermore, the energy efficient optical communication system design using FPGA to produce green optical communication system is also the future demand of high-speed communication. This research will be helpful to develop the high-speed communication system for future generation network.

\section{Acknowledgement}

We are thankful to Universiti Tun Hussein Onn Malaysia (UTHM), Malaysia that encourages us to contribute in research. This work is supported by Research Acculturation Collaborative Effort (RACE) Grant [vot1437] \& Postgraduates Incentive Grant (GIPS) UTHM.

\section{References}

[1] Matsumoto, M., All-optical DQPSK signal regeneration using $2 \mathrm{R}$ amplitude regenerators. Optics Express, 2010. 18(1): p. 10-24. 
[2] Shah, N.S.M., M. Sato, and M. Matsumoto, Adaptive delay control for time-interleaved multi-channel amplitude limiter based on saturation of four-wave mixing in a fiber. Optics Express, 2011. 19(22): p. 21246-

[3] D.J.Richardson, "Suppression of gain variation in a PSA-based phase regenerator using an additional harmonic IEEE Photonics Technology Letters 2014 Vol.26(20) pp.2074-2077.

[4] Roethlingshoefer, T., "All-Optical Multilevel Amplitude and Phase Regeneration" in Photonics Society Summer Topical Meeting Series, 2014 IEEE. 2014, pp. 89-90.

[5] $\mathrm{Yu}, \mathrm{C}$., "Wavelength-shift-free $3 \mathrm{R}$ regenerator for $40-\mathrm{Gb} / \mathrm{s} \mathrm{RZ}$ system by optical parametric amplification in fiber". IEEE photonics technology letters, 2014. 18(21-24): pp. 2569-2571.

[6] Lian, J., et al., All-optical non-conjugated wavelength multicasting of QPSK signal with capability of phase regeneration. Optics express, 2014. 22(19): p. 22996-23006.

[7] Slavík, R., et al., All-optical phase and amplitude regenerator for next-generation telecommunications systems. Nature Photonics, 2010. 4(10): p. 690-695.

[8] Ettabib, M.A., et al. All-Optical Phase Regeneration in a Highly Nonlinear Lead-Silicate Fiber. in Advanced Photonics Congress. 2012. Colorado Springs, Colorado: Optical Society of America, Vol. 20, No. 24 / OPTICS EXPRESS pp. 27212-27220.

[9] Parmigiani, F., et al. All-Optical Phase Regeneration of 40Gbit/s DPSK Signals in a Black-Box Phase Sensitive Amplifier. in National Fiber Optic Engineers Conference. 2010. San Diego, California: Optical Society of America, pp.690-695.

[10] Kakande, J., et al. All-Optical Processing of Multi-level Phase Shift Keyed Signals. in Optical Fiber Communication Conference. 2012. Los Angeles, California: Optical Society of America, pp. OW1I-3.

[11] Willner, A.E., "All-optical signal processing”. Journal of Lightwave Technology, 2014. 32(4): p. 660680.

[12] Marhic, G.K.P.L.a.M.E., Amplification of 800 Gb/s PDM-DPSK Signals in a One-Pump Fiber Optical Parametric Amplifier. In Optical Communication (ECOC), 2014 European Conference on (pp. 1-3). IEEE

[13] Mohd Shah, N.S, "Analysis and experiment of all-optical time-interleaved multi-channel regeneration based on higher-order four-wave mixing in a fiber". Optics Communications, 2011. 284 (19): p. 4687 4694.

[14] Lundstrom, C., "Applications of Fiber-Based Phase-Sensitive Parametric Amplifiers" Photonics Society Summer Topical Meeting Series, 2014 IEEE (pp. 93-94).

[15] Olonkins,et al., Binary PolSK to OOK Modulation Format Conversion in Single-Pump FOPA for Optical Access Networks, Fiber Optics in Access Network (FOAN), 4th International Workshop on 2013 (pp. 15-20).

[16] Gruner-Nielsen, L., et al. Brillouin suppressed highly nonlinear fibers. in Optical Communications (ECOC), 2012 38th European Conference and Exhibition, In Optical Communications (ECOC), 2012 38th European Conference and Exhibition on (pp. 1-3). IEEE.

[17] Slavík, R., et al. Chirp reduction and on/off contrast enhancement via optical injection locking and coherent carrier manipulation, In SPIE Optics+ Optoelectronics (pp. 87810G-87810G). International Society for Optics and Photonics 2013.

[18] Andrekson, "Fiber optical parametric amplifiers in optical communication systems", Laser \& Photonics Reviews, (2015), 9(1), pp. 50-74. 